\title{
How to integrate medical oncology and palliative care?
}

\author{
Alexandru C Grigorescu ${ }^{1 *}$ and Teodorescu Laura Mihaela ${ }^{2}$ \\ ${ }^{1}$ Institute of Oncology Bucharest, Romania \\ ${ }^{2}$ Centrul de Diagnostic si Tratament V. Babes, Romania
}

If we start the discussion from the consideration that there are two modalities to approach the treatment for a patient, we will understand the need to integrate these approaches. The essential aspects of the integration of medical oncology with palliative care are summarized in a very concise manner in an article by Kaasa et al. published in Lancet Oncology in 2018. "The full integration of oncology and palliative care relies on the specific knowledge and skills of two modes of care: the tumour-directed approach, the main focus of which is on treating the disease; and the host-directed approach, which focuses on the patient with the disease" [1].

The Palliative Care Working Group (PCWG) of The European Society of Medical Oncology (ESMO) initiated the accreditation program of some centers integrating medical oncology with palliative care. Currently, due to the large number of centers that have addressed the integration of medical oncology with palliative care, with a personalized approach of the patient in the context of his disease, the ESMO group has taken the name of Designed Centers Working Group (ESMO-DCWG).

In these centers, the fulfillments of the 13 items for accreditation were done differently. ESMO-DCWG accredited these centers even though the resolution of the problems involved in the accreditation requirements was different from the prototype envisaged, by the ideal model developed by the Group, in the idea of promoting integration and broach the importance of palliative care for both medical units, as well as for health decision makers. For example, the ESMO criteria which reflect the issues of integration (Items 1-2 of the thirteen items) are approached differently in different centers: the criteria number 1 ("The center is a cancer center or oncology department which provides closely integrated oncology and palliative care clinical services") has several types of solutions. In some centers, including the "Prof. Dr. Al. Trestioreanu" Institute of Oncology from Bucharest, the palliative beds are located in the medical oncology department and not separately, the same nurses who put the infusions with cytostatics also dealing with palliative care. Other centers, such as Gustave Roussy Institute and many others, have a separate department for supportive-palliative care [2].

The main types of centers that applied for accreditation and obtained it were as follows:

1. Comprehensive centers for cancer treatment (such as major centers in European capitals).

2. Hematology centers that require strict and often long-term surveillance of patients.

3. Centers of specific cancer treatment, for example lung cancer.

4. Hospices in collaboration with the department of medical oncology.

5. Medical oncology departments in collaboration with hospices.
There are differences regarding the organization of medical care from one country to another in Europe and throughout the world. Thus, in Europe there are countries that do not have home care services, such as Greece. There are countries which have hospices and others that don't have this type of care units. The differences between centers are also due to cultural and financing differences of the medical systems between different countries. These differences somehow separate the Western Europe centers from Eastern Europe centers, as well as the countries of Europe from those of the Middle East (except Israel) and the Far East. These cultural and religious differences also influence the practice of palliative care, especially the communication with the patient and the family.

In a review by David Hui et al., there were detected a number of essential indicators of integration which may facilitate the assessment of the level of integration of oncology and palliative care, and may help stakeholders to assess the integration of health providers [3]. This items should be interpreted in the context of the aforementioned differences. These indicators are divided into five broad categories: structure of clinical programs; processes of clinical programs; education; research; administration. In general, the ESMO criteria can be found in the criteria of the David Hui review, but there are also other aspects of integration, such as "national standards/policy" (the $33^{\text {rd }}$ indicator). This element is very important in my opinion, because palliative care is part of the human rights and should be included in the state policy [4]. An important study which revealed the role of integration was the INTEGRATE Project. This study analyzed the benefits of early palliative care implementation in real-world settings. The INTEGRATE Project was a three-year real-world study that promoted the early identification and support of patients with cancer who needed palliative care. This study assessed the feasibility, the stakeholder experiences and the early impact of integration. The results confirmed that early palliative care integration improved the provider's confidence to deliver palliative care and to initiate the Advanced Care Planning (ACP) conversation. In conclusion, a standardized model for the early introduction of palliative care for cancer patients can be integrated into the routine practice of medical oncology services. It requires appropriate education, the integration of palliative care into existing clinical workflows, and support decision makers in health [5].

${ }^{*}$ Correspondence to: Alexandru C. Grigorescu, Institute of Oncology Bucharest, Sos. Fundeni 252, Bucharest, Romania 022328, E-mail: alexgrigorescu2004@ yahoo.com

Received: August 09, 2019; Accepted: October 23, 2019; Published: October 26,2019 


\section{Conclusions}

The palliative care process imply the identification of the patient's overall conditions, including physical (symptoms), psycho-social and spiritual issues, as well as the evaluation of the services provided to him. It is obvious that early integration of palliative care improves the quality of life of patients with a terminally ill condition.

The early adaptation of palliative care is advisable for the management of patients with cancer, and for some localization (lung cancer) there was demonstrated a gain in survival. Other studies for other localization of cancer are also needed.

The integration of palliative care with medical oncology is beneficial for patients and should be included in the therapeutic guidelines, in my opinion.

In Romania, efforts must be done for learning or improving teamwork, as well as for the creation of a palliative care network. This approach cannot be left to chance and must be supported by health decision makers.

Finally, cost-benefit studies should be initiated to assess the integration from this point of view.

\section{References}

1. Kaasa S, Loge JH, Albreht T, Bruera E, Caraceni AT, et al. (2018) Integration of oncology and palliative care. Annals of Oncology 29.

2. https://www.gustaveroussy.fr/en/soins-de-support-0

3. Hui D, Kim YJ, Park JC, Zhang Y, Strasser F, et al. (2015) Integration of oncology and palliative care: a systematic review. Oncologist 20: 77-83. [Crossref]

4. https://www.who.int/news-room/fact-sheets/detail/palliative-care

5. Evans JM, Mackinnon M, Pereira J, Earle CC, Gagnon B, et al. (2019) Integrating early palliative care into routine practice for patients with cancer: A mixed methods evaluation of the INTEGRATE Project.

Copyright: (C2019 Grigorescu AC. This is an open-access article distributed under the terms of the Creative Commons Attribution License, which permits unrestricted use, distribution, and reproduction in any medium, provided the original author and source are credited. 